\title{
论 文
}

\section{高速铁路轨面粗粘度测量方法与分析}

\author{
尹镪, 蔡成标, 朱胜阳* \\ 西南交通大学牵引动力国家重点实验室, 成都 610031 \\ *E-mail: syzhu@swjtu.edu.cn \\ 收稿日期: 2018-02-26; 接受日期: 2018-06-07; 网络版发表日期: 2018-08-01 \\ 国家自然科学基金(批准号: 51708457, 11790283, 51778194)和四川省科技计划(编号: 2017GZ0088, 2017JY0215)资助项目
}

摘要分别采用间接法和直接法对高速铁路无砟轨道钢轨表面粗䊁度进行了测量和分析. 轨面粗鋉度的间接测 量法采用现场实测的钢轨加速度、钢轨振动纵向衰减率, 并结合轮轨动力学计算得到的粗䊁度-钢轨垂向加速度 传递函数，推导得出轨面粗䊁度 $1 / 3$ 倍频程谱. 轨面粗䊅度直接测量法采用钢轨表面粗糙度仪对高速铁路钢轨表 面进行直接检测，并对实测数据进行趋势项去除、异常值去除与时间-频率变换后得到轨面粗糙度 $1 / 3$ 倍频程谱. 将两种方法所测粗䊁度频谱与欧洲粗䊁度限值谱进行对比，结果表明：两种粗䊁度测量方法均可得到较为可靠 的结果, 所测高速铁路轨面粗糙度在 $0.05 \mathrm{~m}$ 以上波长范围小于国外限值，而 $0.05 \mathrm{~m}$ 以下波长范围大于国外限值， 应予以控制。

关键词高速铁路, 粗糙度, 钢轨加速度, 钢轨振动衰减率, 时频转换

\section{1 引言}

轨面粗楉度通常是指波长小于 $1 \mathrm{~m}$ 的短波不平顺 和极短波不平顺(粗糙度), 该波长范围是轮轨系统高 频振动的主要诱因. 在高速铁路运营中会产生几十到 几千赫兹的振动, 该频率范围的振动会导致轮轨噪声, 桥梁结构噪声以及钢轨、轨下胶垫等轨道部件的疲劳 破坏 ${ }^{[1]}$.

目前国内外对铁路轨道长波不平顺的研究较为广 泛和深入. 比较典型的有美国联邦铁路管理局(FRA) 根据大量实测数据得到的美国轨道谱 ${ }^{[2]}$ ，其波长范围 涵盖1.524 304.8 m. 文献[3]提出的德国低干扰和高干 扰谱分别适用于 $250 \mathrm{~km} / \mathrm{h}$ 以上德国高速铁路和
$250 \mathrm{~km} / \mathrm{h}$ 以下普通铁路. 在国内, 20 世纪 70 年代长沙铁 道学院给出了一级干线铁路的轨道不平顺谱表达式 ${ }^{[4]}$. 之后铁道部科学研究院于1999年又提出了中国三大干 线谱 ${ }^{[5]}$. 2007年中国铁道科学研究院在我国既有线进 行了6次大规模提速的背景下提出了中国既有线轨道 谱 $^{[6]}$. 2012年10月, 中国铁道科学研究院以分段曲线拟 合得到了我国300 350 km/h速度等级高速铁路无砟轨 道不平顺谱, 并以此拟定了我国《高速铁路无砟轨道 不平顺谱》铁道行业标准 ${ }^{[7]}$.

近年来随着高速铁路的迅猛发展, 铁路高频振 动、噪声引起极大关注, 然而对轨道短波不平顺(如轨 面粗䊁度)的研究却不多见. EN13231-3:2006《第三部 分：现场钢轨打磨、铣磨和轨道计划作业验收标

引用格式: 尹镪, 蔡成标, 朱胜阳. 高速铁路轨面粗粘度测量方法与分析. 中国科学: 技术科学, 2018, 48: 950-958 Yin Q, Cai C B, Zhu S Y. Measuring method and analysis of rail roughness in high-speed railway (in Chinese). Sci Sin Tech, 2018, 48: 950-958, doi: 10.1360/N092017-00288 
准》 ${ }^{88]}$ 中规定从 4 个波长范围对打磨作业后钢轨轨面 纵向不平顺进行评价验收 (见表 1 ). 其中将 $10 \mathrm{~mm}$ $1000 \mathrm{~m}$ 波长范围分为4段进行分段评价, 评价指标为 峰峰值移动平均值和均方根值移动平均值.

欧洲标准ISO3095 $5^{[9]}$ 中采用TWINS噪声计算软件, 并基于欧洲铁路轮轨测试数据分析了速度 50 $160 \mathrm{~km} / \mathrm{h}$ 的轮轨噪声, 从限制噪声等级出发提出了适 合欧洲的轨面粗粘度 $1 / 3$ 倍频程限值谱, 如图1所示.

相对于 $1 / 3$ 倍频程, 功率谱能反映更完整的频率特 性. 较有代表性的粗䊁度谱有Sato ${ }^{[10]}$ 在研究轮轨高频 振动时采用的法国轨道表面实测谱, 其拟合功率谱公 式如下:

$S(\Omega)=\frac{A}{\Omega^{3}}$,

式中, $S(\Omega)$ 为轨面短波不平顺功率谱密度 $\left(\mathrm{m}^{2} /\left(\mathrm{rad} \mathrm{m}^{-1}\right)\right), \Omega$ 为空间频率 $(\mathrm{rad} / \mathrm{m}), A$ 为短波不平顺 系数, 取值为 $4.15 \times 10^{-8} \sim 5 \times 10^{-7}$.

铁道科学研究院在我国石太(石家庄-太原)线采用 地面测量方式提出了线路垂向短波不平顺谱 ${ }^{[1]}$, 其拟 合公式为

表 1 EN13231-3:2006钢轨打磨验收标准限制

\begin{tabular}{cccc}
\hline 波长范围 $(\mathrm{mm})$ & 采样窗长度 $(\mathrm{m})$ & $\begin{array}{c}\text { 峰峰值移动 } \\
\text { 平均值 }(\mu \mathrm{m})\end{array}$ & $\begin{array}{c}\text { 均方根幅值移动 } \\
\text { 平均值 }(\mu \mathrm{m})\end{array}$ \\
\hline$(10,30]$ & 0.15 & 10 & 4 \\
$(30,100]$ & 0.5 & 10 & 4 \\
$(100,300]$ & 1.5 & 30 & 12 \\
$(300,1000]$ & 5 & 100 & 40 \\
\hline
\end{tabular}

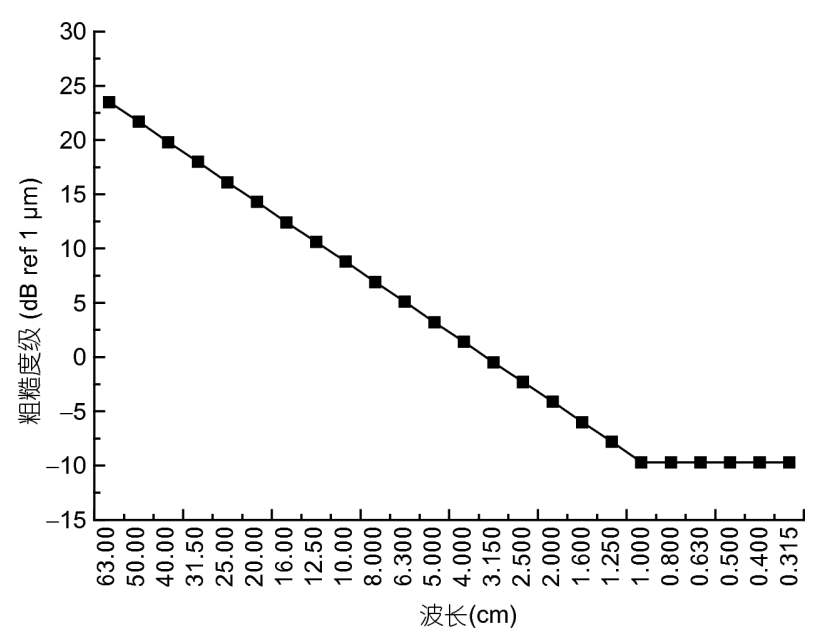

图 1 欧洲轨面粗粘度 $1 / 3$ 倍频程限值谱
$S(f)=0.036 f^{-3.15}$,

式中, $f$ 为空间频率 $\left(\mathrm{m}^{-1}\right)$.

随着2008年京津城际铁路建成并运营，我国成为 首个高速铁路运营速度达到 $350 \mathrm{~km} / \mathrm{h}$ 的国家. 高速铁 路轨道结构主要采用无砟轨道, 与既有线有砟轨道结 构特点存在明显的不同, 国内外原有轨道短波不平顺 谱难以表征高速铁路无砟轨道轨面粗糙度水平. 本文 采用津秦高速铁路现场实测的钢轨垂向加速度、钢轨 振动纵向衰减率, 并结合粗䊁度-钢轨垂向加速度传递 函数, 间接推导得出轨面粗糙度 $1 / 3$ 倍频程谱; 同时, 采 用德国muller-BBM公司的钢轨表面粗粘度仪对轨面 粗糙度进行了直接测量, 将两种方法所测粗粘度频谱 和国外粗糙度限值谱进行了对比分析, 以期为高速铁 路轨面粗糙度检测提供有效的方法.

\section{2 粗楉度间接测量法}

本文首先运用间接测量方法来获取轨面粗楉度, 具体是测量高速列车通过时的钢轨振动加速度、轨道 振动纵向衰减率, 并结合轮轨动力学计算得到的粗粘 度-钢轨垂向加速度传递函数, 推导获得粗粘度 $1 / 3$ 倍 频程谱. 可见, 间接测量方法可获取轮轨联合粗楉度, 但在高速列车运营维护较好的状态下, 钢轨表面粗糙 度占主导地位, 车轮表面粗糙度幅值相对轨面粗粮度 极小, 所以本文在这里认为间接测量法所测结果近似 为轨面粗粘度. 为此, 首先考虑钢轨在简谐激励作用 下的振动情况, 可将距激振力作用点 $x$ 处的钢轨垂向振 动加速度表示为

$A(x)=A_{\text {contact }} \mathrm{e}^{-\beta x}$,

式中, $A_{\text {contact }}$ 为激振点处钢轨垂向振动加速度, $\beta$ 为轨道 振动延纵向衰减因子.

距激振点 $x$ 处钢轨垂向振动加速度谱为

$S_{A}(x)=S_{A_{-} \text {contact }} \mathrm{e}^{-2 \beta x}$,

式中, $S_{A_{\text {_contact }}}$ 为激振点处钢轨垂向振动加速度谱.

当车轮以速度 $V$ 通过, 钢轨加速度振动响应在时 间 $T$ 内平均有 ${ }^{[12,13]}$

$S_{A}^{\text {avg }}=\frac{1}{T} \int_{0}^{T} S_{A_{-} \text {contact }} \mathrm{e}^{-2 \beta V t} \mathrm{~d} t=\frac{1-\mathrm{e}^{-2 \beta V T}}{2 \beta V} S_{A_{-} \text {contact }}$.

当考虑整列车通过时, 测点处钢轨加速度的平均 
响应为

$S_{A}^{\text {avg }}=N \frac{1-\mathrm{e}^{-2 \beta V T}}{2 \beta V} S_{A_{-} \text {contact }}$.

则有

$S_{A}^{\mathrm{avg}} \approx \frac{N}{2 \beta V} S_{A_{-} \text {contact }}$,

式中, $N$ 为轮轴轴数.

假设单车轮定点作用下接触点处粗糙度-钢轨加 速度传递函数为 $H_{\mathrm{r}}(\omega)$, 则 $S_{A_{-} \text {contact }}$ 可表示为

$S_{A_{-} \text {contact }}=H_{\mathrm{r}}^{2}(\omega) \cdot S_{\text {roug }}(\omega)$.

将式(8)代入式(7)可得:

$S_{A}^{\mathrm{avg}}=\frac{N}{2 \beta V} H_{\mathrm{r}}^{2}(\omega) \cdot S_{\text {roug }}(\omega)$.

因此, 粗糙度谱可表示为

$S_{\text {roug }}(\omega)=\frac{S_{A}^{\text {avg }} \cdot 2 \beta V}{N \cdot H_{\mathrm{r}}^{2}(\omega)}$

由式(10)可知, 在已知 $S_{A}^{\mathrm{avg}}, \beta$ 和 $H_{\mathrm{r}}^{2}(\omega)$ 的条件下即 可计算得到粗糙度谱. 下面将分别对这些参量进行求 解与分析.

\section{1 钢轨加速度平均谱}

本文计算中通过现场实测来得到列车通过时钢轨 加速度平均谱. 现场测试条件为 16 节CRH380A型重联 编组列车通过CRTS II 型板式无砟轨道桥梁段, 测试速 度范围为 $200 \sim 350 \mathrm{~km} / \mathrm{h}$, 测试共得到 32 趟有效数据. 试 验采用美国Dytran3145A2, 其量程为 $1000 g$, 测试中采 样频率为 $10000 \mathrm{~Hz}$.

如图2所示, 将5个钢轨加速度传感器等间距布置
于同一块轨道板对应的钢轨跨中. 根据式(11), 对每趟 列车通过时所得的 5 个振动加速度分别进行傅里叶变 换得到各自加速度谱后, 再进行平均得到平均钢轨加 速度平均谱.

$\mathrm{S}_{A}^{\mathrm{avg}}(f)=\frac{\operatorname{FFT}[a(t)]}{2 N_{\mathrm{FFT}} \cdot f_{s}}$

式中, FFT表示傅里叶变换函数, $a(t)$ 为钢轨加速度时 域信号, $N_{\mathrm{FFT}}$ 为信号长度, $f_{s}$ 为采样频率.

以某一趟速度为 $240 \mathrm{~km} / \mathrm{h}$ 列车为例, (1)号位加速 度时域信号如图3(a)所示, 由式(11)可得加速度功率谱 如图3(b)所示. 由图可知, 该行车条件下钢轨垂向加速 度最大值约为 $130 \mathrm{~g}$, 平均值为 $70 \mathrm{~g}$; 加速度功率谱密度 幅值大体上随着频率的升高而呈现逐渐降低的趋势.

\section{2 钢轨垂向加速度-粗粘度传递函数}

理论与试验结果均表明，仅考虑车辆簧下非悬挂 质量(即轮对)与轨道结构的相互作用已足以描述轮轨 高频振动特性以及声辐射特性 ${ }^{[14]}$. 本文建立了考虑车 轮的弹性的轮轨耦合频域分析模型, 如图4所示.

轮对动力学方程可表示为

$$
\mathbf{M}_{\mathrm{w}} \ddot{\mathbf{X}}_{\mathrm{w}}+\mathbf{C}_{\mathrm{w}} \dot{\mathbf{X}}_{\mathrm{w}}+\mathbf{K}_{\mathrm{w}} \mathbf{X}_{\mathrm{w}}=\mathbf{F}_{\text {contact }}+\mathbf{M}_{\mathrm{w}} g
$$

式中, $\mathbf{M}_{\mathrm{w}}, \mathbf{C}_{\mathrm{w}}$ 和 $\mathbf{K}_{\mathrm{w}}$ 分别表示为车轮的质量、阻尼和刚 度矩阵, $\mathbf{X}_{\mathrm{w}}, \dot{\mathbf{X}}_{\mathrm{w}}$ 和 $\ddot{\mathbf{X}}_{\mathrm{w}}$ 分别表示为车轮振动位移、速度 和加速度向量, $\mathbf{F}_{\text {contact }}$ 为车轮受到的荷载向量.

在有限元分析软件ANSYS 中建立轮对有限元模 型，采用八节点实体单元Solid45单元离散，网格限度 为 $8 \mathrm{~mm}$ 可以满足 $6000 \mathrm{~Hz}$ 以下的振动计算. 通过有限 元分析提取车轮振动模态 $10000 \mathrm{~Hz}$ 以内模态特征向 量 $\boldsymbol{\Phi}=\left[\Phi_{1}, \Phi_{2} \cdots \Phi_{M}\right]$ 再运用模态向量的正交性和模
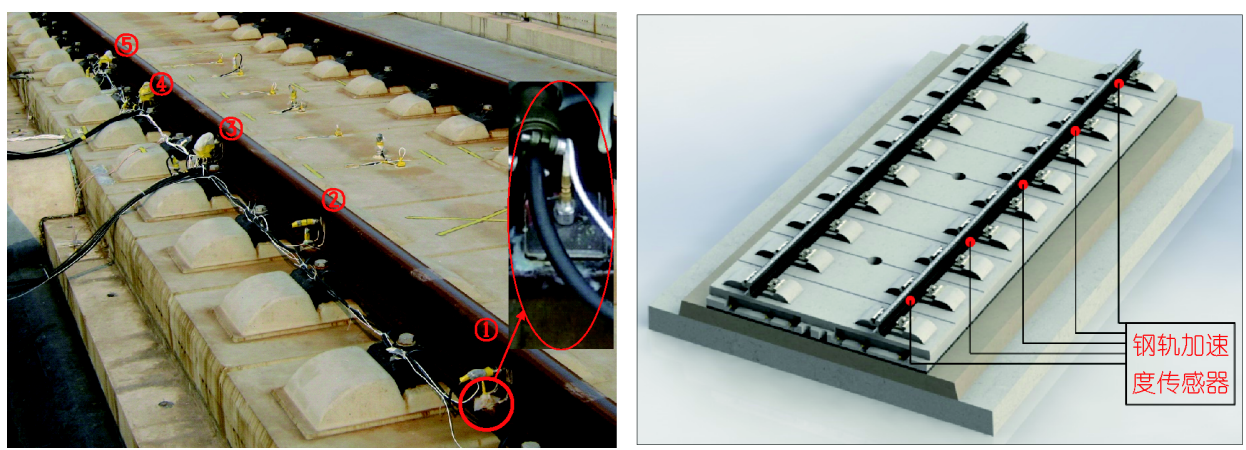

图 2 (网络版彩图)钢轨振动加速度布置图. (a) 现场加速度布置图; (b) 加速度布置示意图 

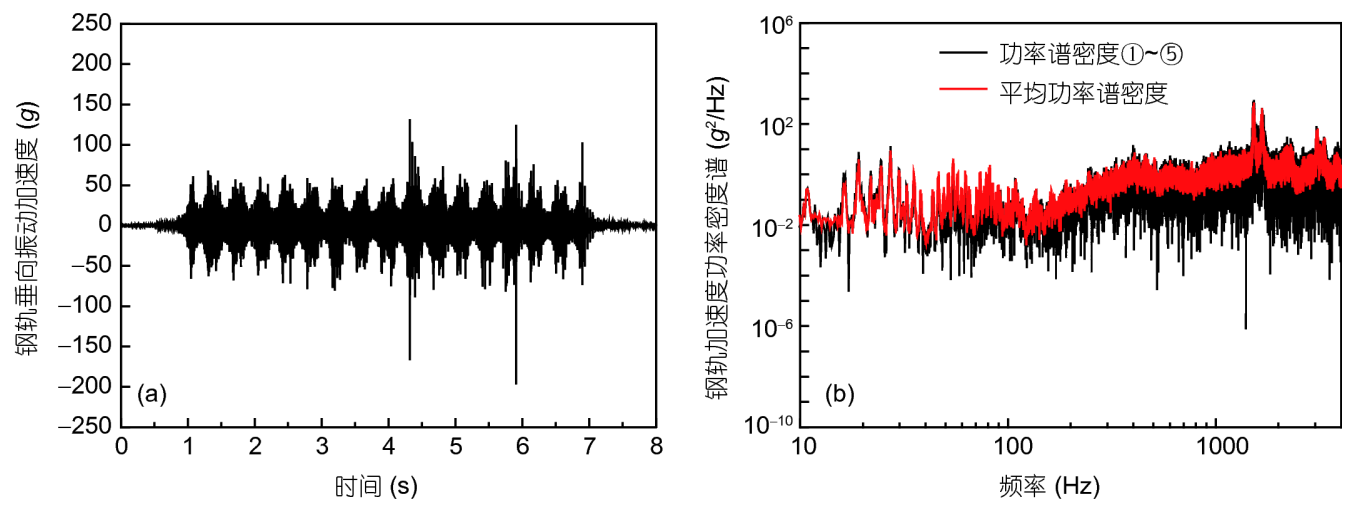

图 3 (网络版彩图)钢轨垂向振动加速度. (a) 加速度时间历程; (b) 加速度功率谱

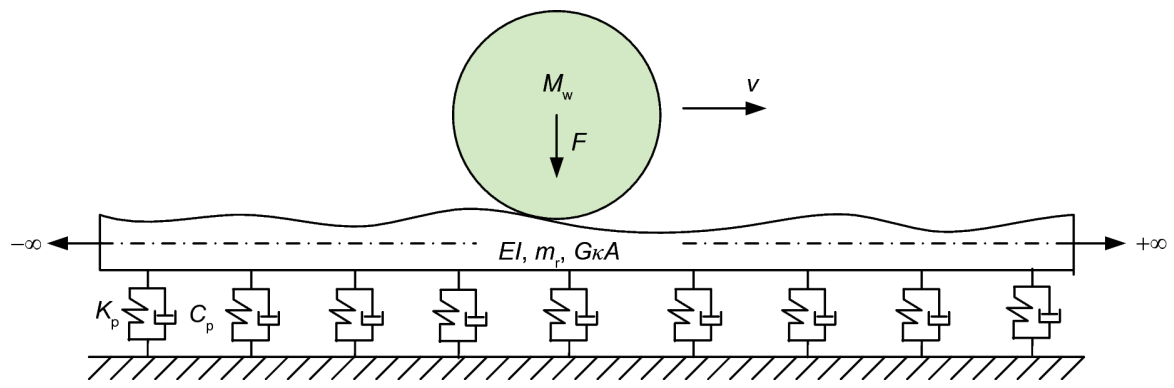

图 4 (网络版彩图) 轮对钢轨耦合模型

态叠加法原理可将式(12)在模态坐标系中解耦为

$\ddot{\mathbf{Q}}_{\mathrm{w}}+2[\xi][\omega] \dot{\mathbf{Q}}_{\mathrm{w}}+\left[\omega^{2}\right] \mathbf{Q}_{\mathrm{w}}=\boldsymbol{\Phi}^{\mathrm{T}} \mathbf{P}$,

其中,

$\boldsymbol{\Phi}^{\mathrm{T}} \mathbf{M}_{\mathrm{w}} \boldsymbol{\Phi}=\mathbf{I}$,

$\boldsymbol{\Phi}^{\mathrm{T}} \mathbf{C}_{\mathrm{w}} \boldsymbol{\Phi}=2[\xi][\omega]$,

$\boldsymbol{\Phi}^{\mathrm{T}} \mathbf{K}_{\mathrm{w}} \boldsymbol{\Phi}=\left[\omega^{2}\right]$,

$\boldsymbol{\Phi}^{T} \mathbf{F}_{\text {contact }}+\boldsymbol{\Phi}^{T} \mathbf{M}_{\mathrm{w}} g=\mathbf{P}$,

$\mathbf{X}_{\mathrm{w}}=\boldsymbol{\Phi} \mathbf{Q}_{\mathrm{w}}$,

式中, I为车轮单位质量矩阵, $\xi$ 为车轮阻尼比, $\omega$ 为车轮 的固有频率.

钢轨采用Timoshenko梁来模拟，其动力学方程可 参考文献[15]. 在频域中进行轮轨动力学计算时, 可将 轮轨接触刚度线性化表示为

$$
\begin{aligned}
K_{h} & =\frac{\partial F_{w r}}{\partial \Delta Z_{w r}}=\left.\frac{3}{2}\left(F_{0}{ }^{2 / 3}+\frac{1}{G} \Delta Z_{w r}\right)^{\frac{1}{2}}\left(\frac{1}{G}\right)\right|_{\Delta Z_{w r} \rightarrow 0} \\
& =\frac{3}{2} \frac{1}{G} F_{0}{ }^{1 / 3},
\end{aligned}
$$

式中, $F_{w r}, \Delta Z_{w r}, F_{0}$ 和 $G$ 分别为轮轨相互作用力、轮轨 相对运动位移、静轮载、轮轨接触常数.

动力学计算中, 车轮采用标称直径为 $920 \mathrm{~mm}$ 整体 钢制车轮，簧下质量为 $2000 \mathrm{~kg}$; 轨道结构参数如表 2 所示.

通过轮轨耦合动力学计算得到单位粗䊁度作用下 钢轨加速度谱, 即钢轨垂向加速度-粗粘度传递函数, 如图5所示.

\section{3 轨道振动衰减率}

通过力锤冲击测试获得无砟轨道振动沿纵向衰减 率，如图6所示，在无砟轨道钢轨顶部布置一加速度传 感器采集钢轨振动加速度, 用力锤坚向敲击传感器附 近点(原点)处, 可分析得到钢轨原点加速度传递函数. 连续敲击 3 次计算得到原点处加速度传递函数平均值, 如图7所示. 同样地, 依次敲击距离传感器1 4个扣件间 距处, 分别得到距激振点不同距离处单位力作用下钢 轨振动加速度 $\mathrm{A}(0), \mathrm{A}(0.625), \mathrm{A}(1.25), \mathrm{A}(1.875)$ 和 $\mathrm{A}(2.5)$.

由钢轨沿纵向振动加速度表达式(3)变换可得: 
表 2 轨道结构参数

\begin{tabular}{cc}
\hline 含义 & 数值 \\
\hline 单位长度钢轨质量 $m_{r}(\mathrm{~kg} / \mathrm{m})$ & 60.64 \\
钢轨密度 $\rho\left(\mathrm{kg} / \mathrm{m}^{3}\right)$ & $7.85 \times 10^{3}$ \\
钢轨截面面积 $A\left(\mathrm{~cm}^{2}\right)$ & 77.45 \\
钢轨截面剪切形状因子 $\kappa$ & 0.5331 \\
钢轨弹性模量 $E(\mathrm{~Pa})$ & $2.059 \times 10^{11}$ \\
钢轨截面惯量 $I\left(\mathrm{~m}^{4}\right)$ & $3.217 \times 10^{-5}$ \\
钢轨剪切模量 $G(\mathrm{~Pa})$ & $7.919 \times 10^{10}$ \\
扣件数 $N$ & 30 \\
扣件间距 $l_{\mathrm{s}}(\mathrm{m})$ & 0.625 \\
轨下胶垫阻尼损失因子 $\eta_{\mathrm{p}}$ & 0.25 \\
轨下胶垫初始刚度 $K_{\mathrm{p}}(\mathrm{N} / \mathrm{m})$ & $4 \times 10^{7}$ \\
\hline
\end{tabular}

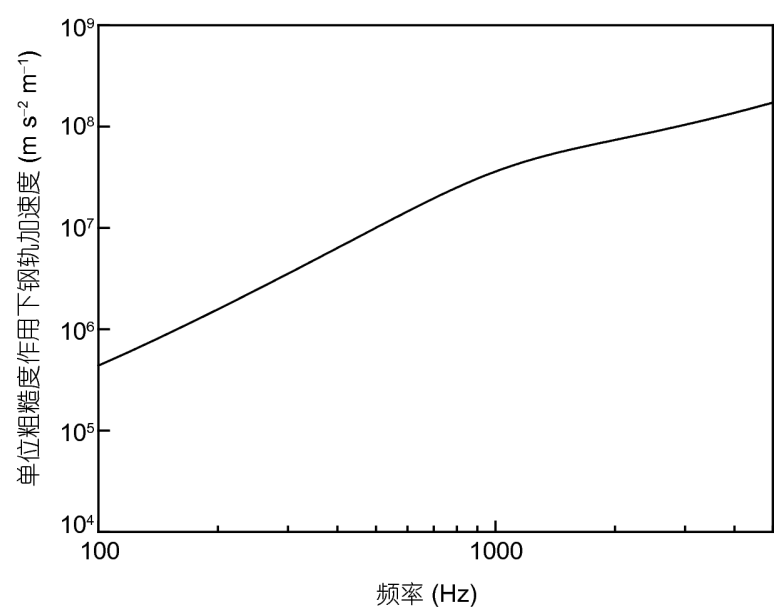

图 5 单位粗楉度作用下钢轨振动加速度

$\mathrm{e}^{-\beta x}=\frac{A(x)}{A_{\text {contact }}} \Rightarrow \beta=-\frac{1}{x} \log _{e}^{A(x) / A_{\text {contact }} .}$

将上述测得的钢轨振动加速度代入式(20), 可得4 组钢轨振动衰减率 $\beta$, 从而取平均值得到轨道衰减率, 如图8所示. 同时, 在图7和8中均给出了仿真计算得到 的钢轨原点加速度传递函数和轨道振动衰减率. 由图 可知，仿真与实测结果吻合较好，证明了本文前述所 建模型的可靠性.

\section{4 间接测量结果}

由上述现场实测得到的钢轨加速度平均谱和轨道 振动衰减率, 结合仿真分析获得的钢轨垂向加速度-粗 䊁度传递函数，采用式(10)即可得到钢轨表面粗粘度

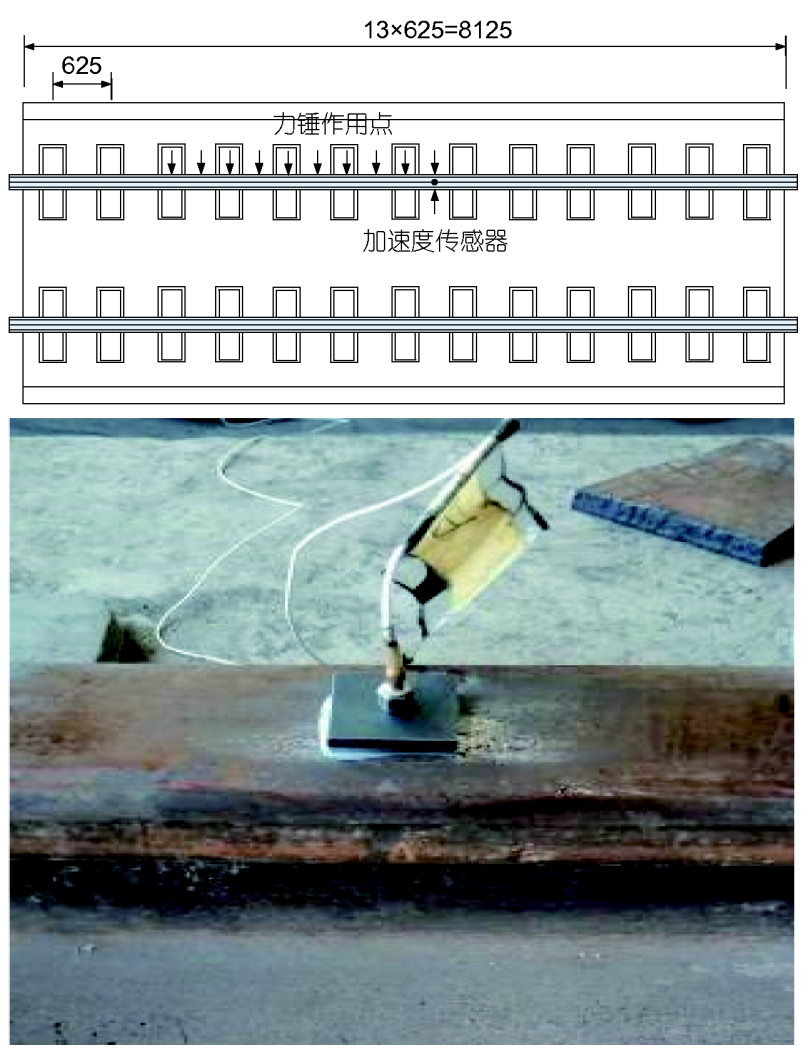

图 6 (网络版彩图)轨道振动沿纵向衰减率测试

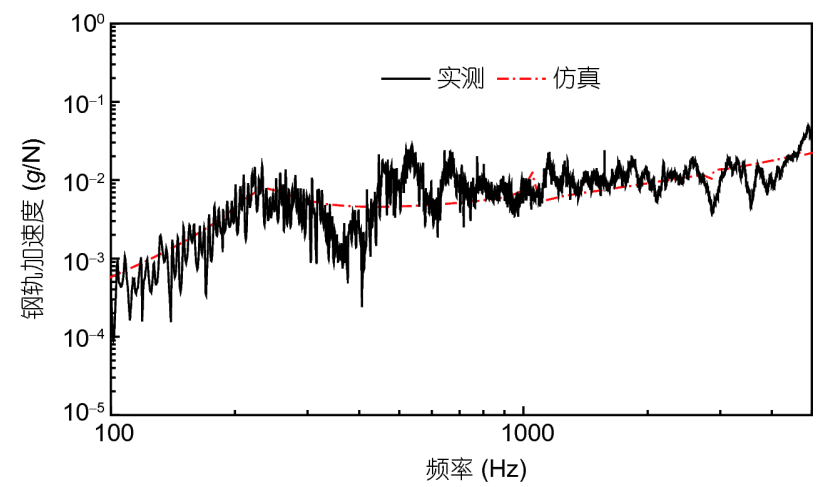

图 7 (网络版彩图)钢轨垂向加速度传递函数

功率谱, 如图9所示.

\section{3 粗粘度直接测量法}

\section{1 现场测试}

为对高速铁路轨面粗粘度进行有效地检测与评 价，采用德国Muller-BBM公司生产的钢轨表面粗糙度 


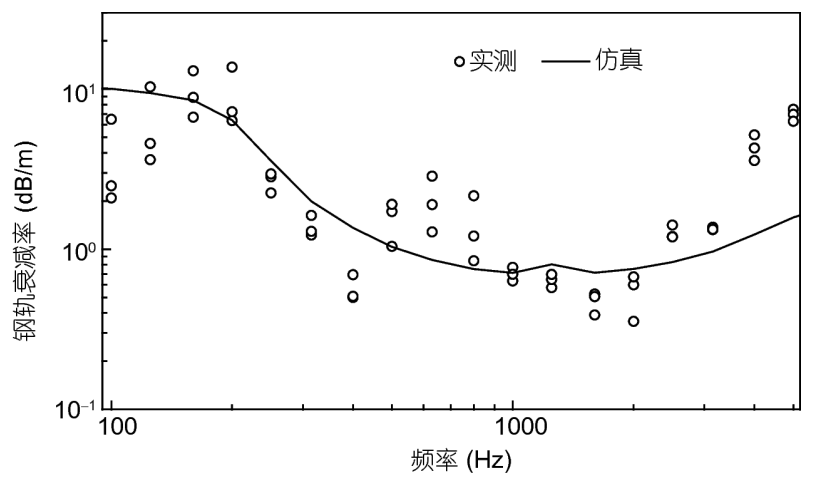

图 8 钢轨振动衰减率

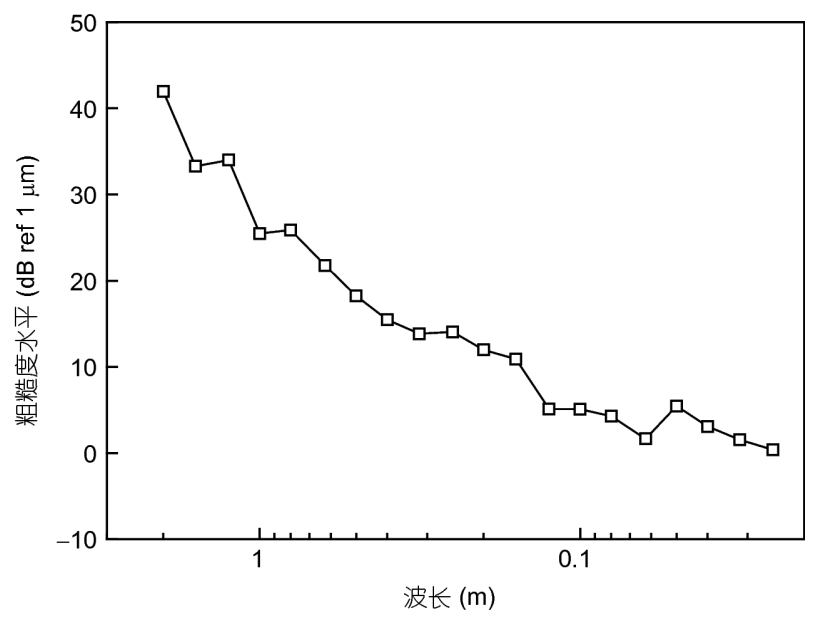

图 9 钢轨表面粗糙度功率谱

仪对津秦高速铁路进行了直接测量, 该仪器为非接触 式采样, 采样间隔为 $0.5 \mathrm{~mm}$, 单次测量里程为 $1.2 \mathrm{~m}$. 每次测量均对以测试光带为中心的 3 条相距 $5 \mathrm{~mm}$ 的轨 迹进行测量, 取平均值得到该段轨面粗糙度值. 测试区 段轨道类型为桥上CRTS II 型板式无砟轨道. 图10和11 分别为测试现场和实测轨面粗粘度数据样本.

\section{2 异常值和趋势项去除}

由于外界干扰、传感器和信号传输异常等原因, 实测轨面粗粘度数据往往会包含异常值，同时标定误 差和惯性器件的温度漂移会导致检测数据存在趋势 项. 为了提高轨面粗䊁度的检测精度, 就需要对实测数 据进行预处理, 剔除其中的异常值和趋势项.

高速铁路轨道不平顺变化率一般控制到 $1 \%$, 实测 数据统计表明正常的轨道不平顺变化率一般不会大于 $3 \%$, 超过 $3 \%$ 可认为是异常值. 由此本文参考文献 $[16]$

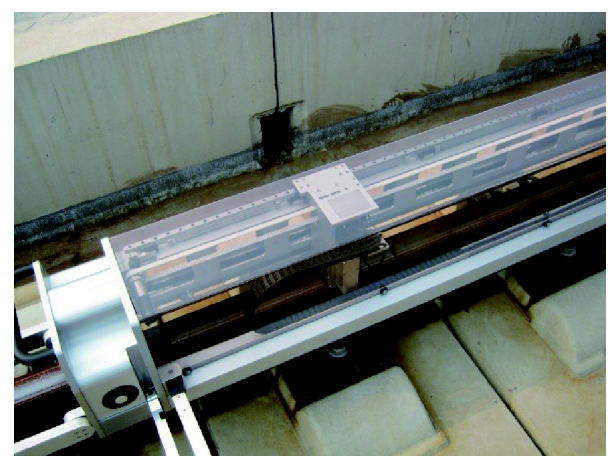

图 10 (网络版彩图)测试现场

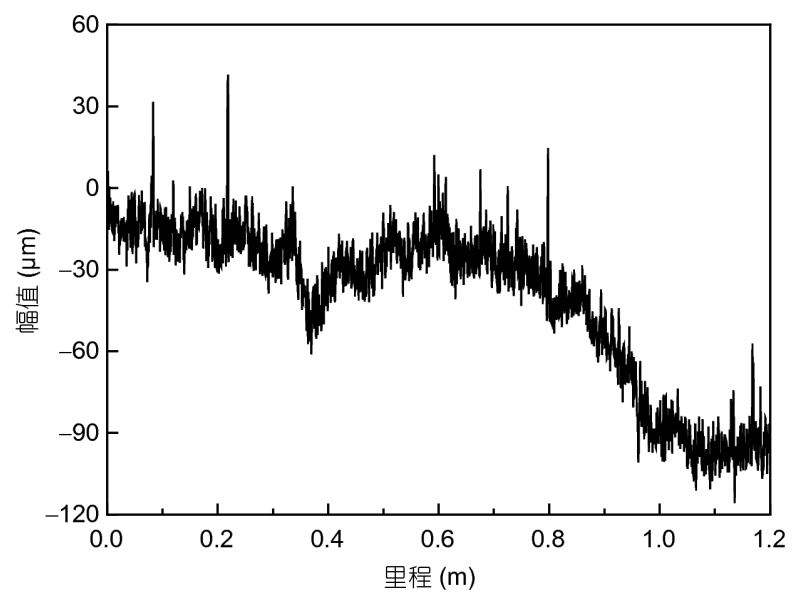

图 11 轨面粗䊁度数据样本

所提出的两种轨道不平顺数据异常值判断和处理的工 程快速算法: (1) 如果相邻两点变化率大于 $3 \%$, 按 $1 \%$ 变化率处理; (2) 如果任意两点中间位置的轨道不平顺 幅值减去该两点不平顺幅值的均值除以该两点距离的 一半大于 $3 \%$, 则利用该两点幅值对两点之间的数据进 行线性插值处理.

图12为去除异常值后的轨面粗糙度样本，由波长一 幅值关系图可知，毛刺的存在对粗粘度的影响主要在 极短波长部分, 而长波长幅值几乎不受其影响.

由于单次测量里程较短, 数据截断会使长波趋势 蔓延到短波长区域. 文中采用最小二乘法拟合出一条 直线及趋势项，将原始数据与之相减达到去趋势项的 结果, 如图13所示.

\section{3 频谱分析}

对实测数据进行时频分析得到轨面粗糙度 $1 / 3$ 倍 频程谱, 如图14所示. 由图可知, 轨面粗粘度幅值随着 

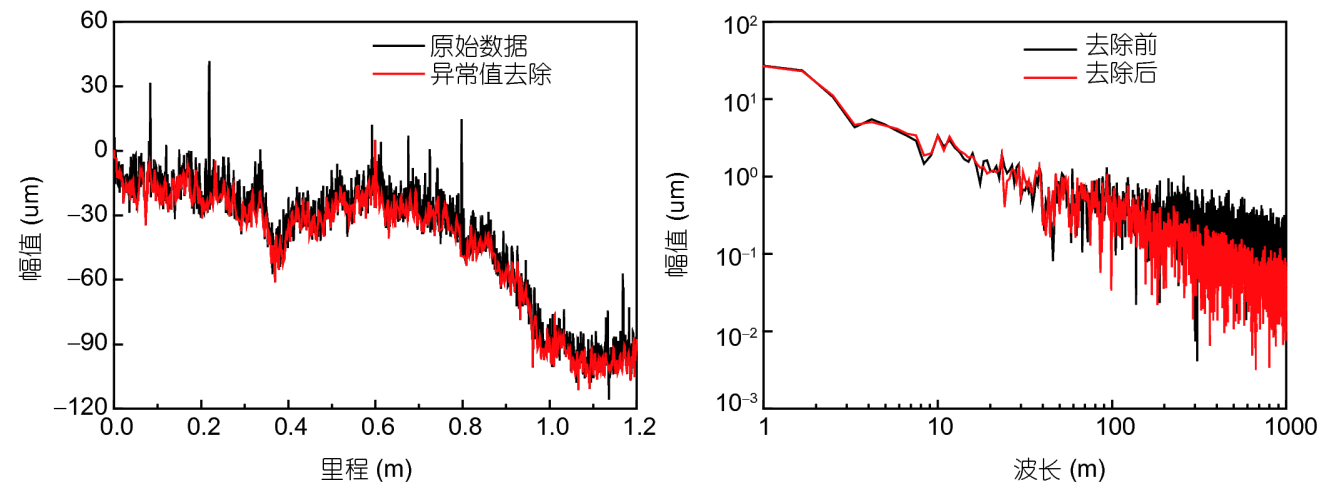

图 12 (网络版彩图)异常值去除后的轨面粗糙度样本. (a) 里程-幅值关系图; (b) 波长-幅值关系图

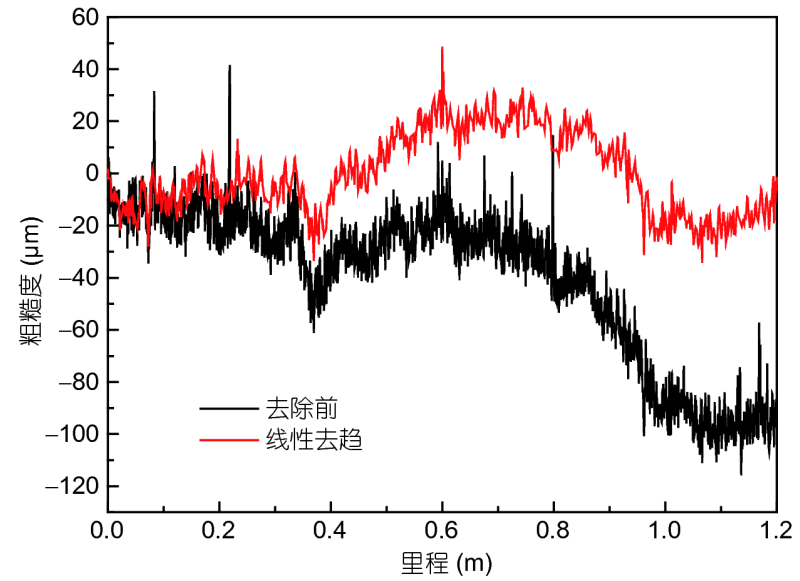

图 13 (网络版彩图)粗粘度趋势项去除

波长的减小而下降.

\section{4 短波不平顺比较}

图15给出了采用两种测量方法得到的无砟轨道钢 轨表面粗楉度谱以及欧洲标准ISO3095中规定的轨面 粗䊁度标准.

由图可知，基于现场实测的钢轨垂向加速度、钢 轨振动纵向衰减率, 并结合粗粘度-钢轨垂向加速度传 递函数，推导得出轨面粗糙度水平与采用钢轨粗糙度 仪直接测量的粗糙度幅值及趋势较为相似，可见由钢 轨加速度间接分析得到的轨面粗粘度具有一定的准确 性和可靠度.

与欧洲ISO: 3095标准对比可知，该高速铁路轨面 不平顺在 $0.05 \mathrm{~m}$ 波长范围以上部分满足限值要求，而 在 $0.05 \mathrm{~m}$ 以下波长部分超出了该标准规定的限值，应 予以振动与噪声计算控制.

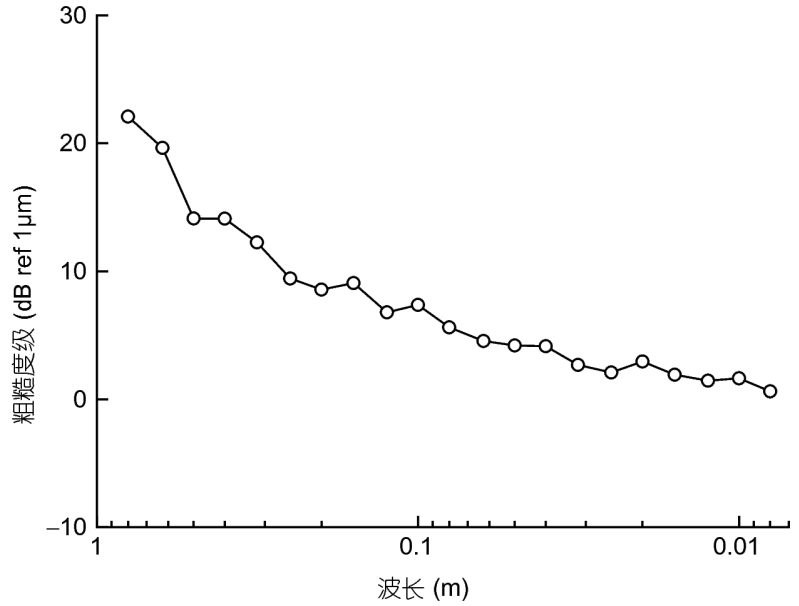

图 14 粗糙度 $1 / 3$ 倍频程

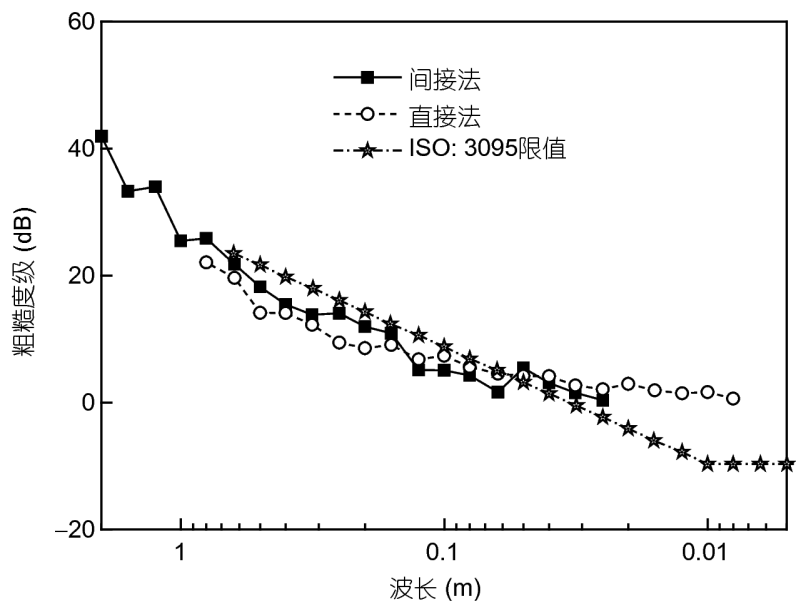

图 15 粗粘度幅值对比

\section{4 结论}

为了获取高速铁路轨面粗糙度谱, 通过现场实测 
得到了高速列车通过时钢轨加速度平均谱, 再运用建 立的考虑车轮的弹性的轮轨耦合频域分析模型，计算 得到了粗鋉度-钢轨垂向加速度传递函数, 并结合现场 力锤冲击试验获得的轨道振动沿纵向衰减率，间接预 测出轨面粗粘度 $1 / 3$ 倍频程谱. 同时, 采用钢轨表面粗 楉度仪对进行了直接检测, 并对测试数据进行趋势项
和异常值去除、时频变换后得到轨面粗糙度 $1 / 3$ 倍频 程谱. 对比结果表明两种测量方法均可获得较为可靠 的结果，可以有效应用于粗粘度的测量. 与欧洲粗粘 度限值谱对比可知, 该高速铁路轨面不平顺在 $0.05 \mathrm{~m}$ 波长范围以上部分满足限值要求, 而在 $0.05 \mathrm{~m}$ 以下波 长部分超出了该标准规定的限值, 应予以控制.

\section{参考文献}

1 Knothe K, Grassie S L. Modelling of railway track and vehicle/track interaction at high frequencies. Vehicle Syst Dyn, 1993, 22: 209-262

2 Humid A, Rasnysseb K, Baluja M, et al. Analytical descriptions of track geometry variations: Volume I \& II. Technical Report. USA: ENSCO Inc., 1983

3 德国联邦铁路慕尼黑研究中心. 城间特快列车ICE技术任务书. 成都: 西南交通大学出版社, 1993

4 长沙铁道学院随机振动研究室. 关于机车车辆/轨道系统随机激励函数的研究. 长沙铁道学院学报, 1985, 1: 1-36

5 铁道部科学研究院. 我国干线轨道不平顺功率谱的研究. 技术报告. 北京: 铁道部科学研究院, 1999

6 中国铁道科学研究院. 既有线轨道不平顺谱的研究. 技术报告. 北京: 中国铁道科学研究院, 2007

7 国家铁路局. TN/T3352-2014高速铁路无砟轨道不平顺谱. 北京: 中国铁道出版社, 2014

8 Technical Committee CEN/TC265. Railway applications-track-acceptance of works-Part 3: Acceptance of rail grinding, milling and planning work in track. BS EN13231-3:2006, British Standards Institution, 2006

9 European Committee for Standardization. BS EN ISO 3095 Railway applications-acoustics-measurement of noise emitted by railbound vehicles. British Standards Institution, 2005

10 Sato Y. Study on high frequency vibration in track operation with high-speed trains. Quart Report, 1977, 18: 22-27

11 王澜. 轨道结构随机振动理论及其在轨道结构减振中的应用. 博士学位论文. 北京: 铁道部科学研究院, 1988

12 Remington P J. Wheel/rail rolling noise, I: Theoretical analysis. J Acoust Soc Am, 1987, 81: 1805-1823

13 雷晓燕, 圣小珍. 铁路交通噪声与振动. 北京: 科学出版社, 2004

14 翟婉明. 铁路轮轨高频随机振动理论解析. 机械工程学报, 1997, 33: 20-25

15 翟婉明. 车辆-轨道耦合动力学. 第4版. 北京: 科学出版社, 2015

16 康熊, 刘秀波, 李红艳, 等. 高速铁路无砟轨道不平顺谱. 中国科学: 技术科学, 2014, 44: 687-696 


\title{
Measuring method and analysis of rail roughness in high-speed railway
}

\author{
YIN Qiang, CAI ChengBiao \& ZHU ShengYang
}

State Key Laboratory of Traction Power, Southwest Jiaotong University, Chengdu 610031, China

The indirect method and direct method are employed to measure and analyze the rail roughness of ballastless tracks in high-speed railway, respectively. The indirect method deduces the one-third octave band spectrum of the rail roughness by using the field tested rail acceleration, longitudinal decay rate of rail vibration, and the transfer function between the rail roughness and rail acceleration obtained by wheel-rail dynamic calculation. The direct method employs the rail roughness measurement instrument to directly measure the rail surface in high-speed railway, and then the one-third octave band spectrum of the rail roughness is acquired after the trend term removal, the outlier removal and the time-frequency transformation of the measured data. The rail roughness spectra obtained by the two methods are compared with European roughness limit spectrum. Results show that reliable results can be obtained by both the indirect and direct methods, the measured rail roughness of the tested high-speed railway is smaller than the European spectrum limit when the wavelength is larger than $0.05 \mathrm{~m}$, whereas it exceeds the European spectrum limit when the wavelength is smaller than $0.05 \mathrm{~m}$, which should be controlled.

high-speed railway, rail roughness, rail acceleration, rail vibration decay rate, time-frequency transformation

doi: $10.1360 /$ N092017-00288 